Methods We performed western blotting for ZEB1, E-cadherin, vimentin and $\alpha$-tubulin to identify the epithelial-mesenchymal status of eight primary HCC cell lines. IHC was undertaken on paraffin sections from 40 patients who underwent resections for primary HCC between May 1997 and November 2010 and scored by two independent pathologists. Clinicopathological data were collated retrospectively and patient survival calculated using the Kaplan-Meier method. We transfected ZEB1 into Huh7 and HepG2 cell lines by electroporation and assessed EMT related changes in cell motility using Boyden chambers (pore size: $8 \mu \mathrm{m}$ ) and serum as chemo-attractant.

Results Western blotting of proteins from eight HCC cell lines demonstrated reciprocal expression of ZEB1 and E-cadherin, suggesting EMT promotes a migratory phenotype in HCC. ZEB1 also significantly increased cell motility as a threefold increase in cell migration was observed after ZEB1 transfection into Huh7 cells $(23 \pm 4$ vs $79 \pm 5)$. ZEB1 positivity was detected in $11 / 40$ specimens analysed by IHC. Statistical analysis highlighted ZEB1 as an independent prognostic marker favouring a significant reduction in cancer specific (41 vs 16 months, $p$

Conclusion Our results suggest that ZEB1 induced EMT promotes tumour progression and metastasis in HCC, and that over-expression of ZEB1 may represent an independent prognostic biomarker in patients with HCC.

Competing interests None declared.

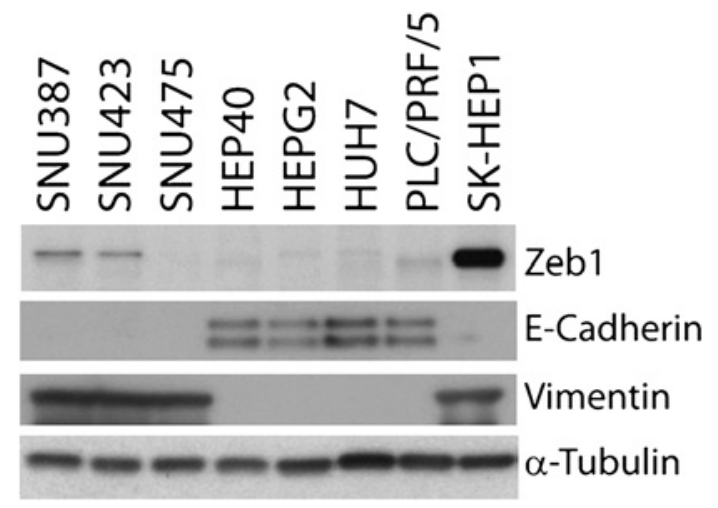

Abstract PM0-135 Figure 1

\section{PM0-136 DEFECTIVE INHIBITORY MOLECULES EXPRESSION MAY CONTRIBUTE TO BREAKDOWN OF TOLERANCE CHARACTERISTIC OF AUTOIMMUNE LIVER DISEASE}

doi:10.1136/gutjnl-2012-302514b.136

${ }^{1,2} \mathrm{R}$ Liberal, ${ }^{*} \mathrm{C}$ Grant, ${ }^{2} \mathrm{C}$ Grant, ${ }^{2} \mathrm{G}$ Mieli-Vergani, ${ }^{2} \mathrm{D}$ Vergani, ${ }^{2} \mathrm{M}$ Longhi. ${ }^{1}$ Faculdade de Medicina da Universidade do Porto, Porto, Portugal; ${ }^{2}$ King's College London, London, UK

Introduction Autoimmune hepatitis (AIH) is a severe hepatopathy often progressing to end-stage liver disease. Evidence implicates the involvement of both CD4 and CD8 T cell responses in its pathogenesis. There are a number of different inhibitory molecules expressed by $\mathrm{T}$ cells that can attenuate $\mathrm{T}$ cell receptor signalling. These include cytotoxic $\mathrm{T}$ lymphocyte antigen-4 (CTLA-4), programmed death-1 (PD-1), and the recently described $\mathrm{T}$ cell immunoglobulin and mucin domain-3 (Tim-3). Whether a disturbed expression of these inhibitory molecules can result in an increased susceptibility to autoimmune liver disease is unknown.

Aims to evaluate the expression of CTLA-4, PD-1, and Tim-3 by CD4 and CD8 T cells in patients with autoimmune hepatitis.

Methods 12 ANA/SMA + AIH patients ( 6 females, median age: 14 years) and 6 healthy subjects (HS, four females, median age:
26.4 years) were studied. Phenotype of CD4 and CD8 T cells was determined by flow cytometry using monoclonal antibodies against CD4, CD8, PD-1 and Tim-3. Expression of CTLA-4 was determined by intracellular staining.

Results The frequency of Tim- $3^{\text {pos }}$ and PD- $1^{\text {pos }}$ cells within CD4 and CD8 $\mathrm{T}$ cells was lower in $\mathrm{AIH}$ (CD4 ${ }^{\text {pos }} \mathrm{Tim}-3^{\text {pos }}$ : $1.6 \pm 0.3$

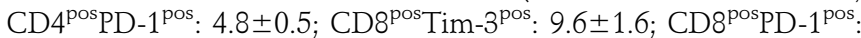
$6.7 \pm 0.7)$ than in $\mathrm{HS}\left(\mathrm{CD} 4^{\text {pos }} \mathrm{Tim}^{-3^{\text {pos }}}{ }^{6} 6.2 \pm 0.8\right.$, PposPD- ${ }^{\text {pos }}$ 8.1 $\pm 1.9, \mathrm{P}=0.04 ; \mathrm{CD}^{\mathrm{pos}} \mathrm{Tim}-3^{\text {pos }}: 15.8 \pm 1.8, \mathrm{P}=0.04 ; \mathrm{CD} 8^{\mathrm{pos}} \mathrm{PD}-$ $1^{\text {pos }}$ : $12.6 \pm 1.46 .7 \pm 0.7$, Ppos cells between the two groups of subjects. While in health dually Tim-3 and PD-1 positive populations are recognisable $\left(\mathrm{CD} 4^{\text {pos }}\right.$ Tim- $3^{\text {pos }} \mathrm{PD}-1^{\text {pos: }}$ : $0.7 \pm 0.1$; CD8 ${ }^{\text {pos }}$ Tim- $3^{\text {pos }}$ PD- $1^{\text {pos }}$ : $\left.1.7 \pm 0.4\right)$, they are reduced in $\mathrm{AIH}$

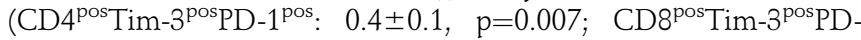

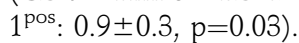

Conclusion AIH patients have fewer $\mathrm{PD}-1$ and Tim 3 positive cells within both CD4 and CD8 T cells. Defective expression of these negative immune-regulatory molecules may contribute to breakdown of tolerance, possibly accounting for the initiation and/or perpetuation of the autoimmune liver attack.

Competing interests None declared.

\section{PM0-137 PHENOTYPIC AND FUNCTIONAL SIGNATURE OF CD4POSCD25HIGHCD127 LOW REGULATORY T-CELLS IN AUTOIMMUNE HEPATITIS}

doi:10.1136/gutjnl-2012-302514b.137

${ }^{1,2} \mathrm{R}$ Liberal, ${ }^{*} \mathrm{C}$ Grant, ${ }^{2} \mathrm{G}$ Mieli-Vergani, ${ }^{2} \mathrm{D}$ Vergani, ${ }^{2} \mathrm{M}$ Longhi. ${ }^{1}$ Faculdade de Medicina da Universidade do Porto, Porto, Portugal; ${ }^{2}$ King's College London, London, UK

Introduction In autoimmune hepatitis $(\mathrm{AIH}) \mathrm{CD} 4{ }^{\text {pos }} \mathrm{CD} 25^{\text {high }}$ regulatory T-cells (T-regs), a subset central to immune-tolerance, are numerically defective and impaired in their ability to control effector cell function. At variance with CD4 effectors, T-regs, classically known as CD25 $5^{\text {high }}$ and FOXP3 ${ }^{\text {pos }}$, express low levels of the activation marker CD127. The aim of the current study was to provide a phenotypic and functional profile of $\mathrm{CD} 4{ }^{\text {pos }} \mathrm{CD} 255^{\text {high }} \mathrm{C}$ D127 $7^{\text {low }}$ T-regs (CD127 ${ }^{\text {low }}$ T-regs) in AIH and to explore to what extent absence or low levels of CD127 impact on T-reg ability to suppress.

Methods $20 \mathrm{ANA} / \mathrm{SMA}+\mathrm{AIH}$ patients and 12 healthy subjects (HS) were studied. T-reg phenotype was determined by flow cytometry using antibodies to CD4, CD25, CD127, CTLA-4 and Galectin-9, a molecule linked to T-reg ability to suppress. T-reg transcription factor and cytokine profile were assessed by intracellular staining. CD127 $7^{\text {low }}$ T-reg ability to suppress was evaluated in a proliferation assay following co-culture with $\mathrm{CD} 25^{\text {neg }}$ target cells.

Results In $\mathrm{AIH}$ CD $4{ }^{\text {pos }} \mathrm{CD} 25^{\text {high }}$ cells contained fewer $\mathrm{CD} 127^{\text {low }}$ cells than in HS. Compared to conventional $\mathrm{CD} 4{ }^{\text {pos }} \mathrm{CD} 25^{\text {high }}$ (cTregs), CD127 $7^{\text {low }}$-regs from both $\mathrm{AIH}$ and $\mathrm{HS}$ had a) higher numbers of FOXP3 ${ }^{\text {pos }}$, CTLA-4 ${ }^{\text {pos }}$, Galectin-9 $9^{\text {pos }}$ and IL-10 ${ }^{\text {pos }}$ cells; $b$ ) lower numbers of T-bet ${ }^{\text {pos }}$ RORC ${ }^{\text {pos }}$, IFNg ${ }^{\text {pos }}$ and IL-17 $7^{\text {pos }}$ cells; and c) similar numbers of $\mathrm{TGF}-\mathrm{b}^{\text {pos }}$ cells. In $\mathrm{AIH}, \mathrm{CD} 127^{\text {low }} \mathrm{T}$-regs contained fewer FOXP3 ${ }^{\text {pos }}$, CTLA-4 ${ }^{\text {pos }}$, Galectin-9pos IL-10 $^{\text {pos }}$ and $\mathrm{TGF}^{\mathrm{p}}{ }^{\text {pos }}$ cells and higher frequencies of T-bet ${ }^{\text {pos }}, \mathrm{RORC}^{\text {pos }}, \mathrm{IFNg}^{\text {pos }}$ and IL-17 ${ }^{\text {pos }}$ cells than in HS. CD127 ${ }^{\text {low }}$ T-regs inhibited CD2 $5^{\text {neg }}$ cell proliferation more effectively than $\mathrm{cT}$-regs, though less markedly in AIH than in HS. In AIH, treatment with anti-IFNg and anti-IL-17 neutralising antibodies ameliorated the suppressive ability of cTregs, while leaving unchanged that of $\mathrm{CD} 127^{\text {low }} \mathrm{T}$-regs; exposure to anti-IL-10 neutralising antibodies reduced cT-reg suppression in HS, but not in AIH.

Conclusion $\mathrm{CD} 127^{\text {low }}$ T-regs bear the phenotypic and functional signature of "true T-regs". Low numbers and reduced suppressive function of CD127 $7^{\text {low }}$-regs in AIH may contribute to breakdown of 